\title{
Repubblica Ceca o Cechia?
}

\author{
Enzo Caffarelli
}

PUBBLICATO: 09 SETTEMBRE 2016

\begin{abstract}
Quesito:
Sono arrivati vari quesiti che riguardano la Repubblica Ceca: alcuni (i più) vertono sulla possibilità di usare in sua vece la più semplice denominazione Cechia; altri chiedono se in questo contesto l'iniziale di Ceca possa essere minuscola; altri ancora se, dopo le preposizioni, è possibile omettere l'articolo (in Repubblica Ceca invece che nella Repubblica (eca); altre domande, infine, riguardano l'etnico ceco e avanzano proposte grafiche o addirittura nuove denominazioni alternative per evitare l'omofonia con cieco, che può causare fastidiosi equivoci.
\end{abstract}

\section{Repubblica Ceca o Cechia?}

Prima di entrare nella questione centrale - Cechia o Repubblica Ceca - rispondo alle domande di carattere fonetico, morfologico e sintattico. L'abitante della nazione slava che ha per capitale Praga è detto ceco. L'introduzione di variazioni come czeco o boemoravo (che peraltro lascerebbe fuori la porzione ceca della Slesia), di conio suggestivo e di buona funzionalità, si scontrano con la difficoltà di imporre a tavolino parole nuove e piuttosto complicate per il parlante italiano.

Né aggiungere un accento grafico aiuterebbe granché, considerato che al fine di differenziare l'etnico ceco dalla voce cieco, che è il vero nodo della nostra lingua a rischio di imbarazzanti fraintendimenti, esiste già - almeno nello scritto - la $i$ dell'originario dittongo, pronunciata solo in alcune varietà regionali. La segnatura dell'accento dovrebbe influenzare il parlante fino al punto di spingerlo a una pronuncia chiusa della e di ceco. Ma già i dialetti e le parlate regionali italiane non concordano sul grado di apertura della vocale tonica di cieco.

Quanto alla scrizione maiuscola o no di Ceca nella denominazione della Repubblica, è caldamente consigliata la maiuscola: non si tratta di mero aggettivo, ma di una parte fondamentale della breve catena onomastica che individua la nazione, anzi lo Stato.

E vero poi che in rete la dizione "in Repubblica Ceca" supera leggermente - a consultare il motore di ricerca Google - l'altra forma "nella Repubblica Ceca"; ma, come segnala una lettrice, davanti a un sostantivo come Repubblica, voce di lessico anche se nel caso usata come nome proprio, l'articolo e dunque la preposizione articolata non dovrebbero venir meno. Dunque "nella Repubblica Ceca", cosi come della, dalla, per la... Repubblica Ceca.

La frequenza di in nel caso ceco è analoga a quella che si riscontra nella Rete per la Repubblica Dominicana; ma tale frequenza è minore per la Repubblica Centrafricana, e ancora più per la Repubblica Democratica del Congo e per la Repubblica di San Marino (e praticamente nulla per la Repubblica Sudafricana); può notarsi come la preposizione semplice, in genere, sia tanto più frequente quanto meno esiste una forma d'uso accorciata dello Stato: l'esistenza dello Stato della Dominica blocca la riduzione di Repubblica Dominicana; Centrafrica è utilizzato poco perché a rischio di confusione con un coronimo di più ampia estensione; Congo è d'uso comune (anche se ciò impedisce la distinzione tra l'ex Congo Belga e l'ex Congo Francese).

Il ricorso a in davanti a Repubblica Ceca (e negli altri casi) sarà frutto di tre cause concomitanti: un primo fattore è diamesico, relativo alla scrittura rapida di notizie e messaggi on line; un secondo è dato dalla pressione analogica degli altri sintagmi contenenti un coronimo ("in Asia, in Francia, in Lombardia..."); un terzo è il crescente uso - specie nel linguaggio della finanza, della politica e di conseguenza giornalistico - della preposizione semplice in davanti a nomi di aziende, enti, associazioni: "in RAI, in Telecom, in Mondadori, in CGIL...”.

Vero è, peraltro, che quando il nome proprio è formato o accompagnato da una voce di lessico, prevale l'uso di anteporre la preposizione articolata: "nella Francia meridionale, nell'ex Jugoslavia, nella Cipro turcofona", ecc. 
Ciò vale a maggior ragione quando si tratti di coronimo stabilizzato, ossia di un nome proprio ufficiale - "negli Stati Uniti, nel Regno Unito, nella Grande Polonia" - ma non senza eccezioni ("in Gran Bretagna").

E veniamo alla possibilità di utilizzare Cechia al posto della dizione ufficiale Repubblica Ceca (in ceco: Česká republika). Ebbene, la nazione è già chiamata nella forma semplice, che esclude "repubblica", in molte lingue del mondo: Tschechien in tedesco; Českáin sloveno, croato, serbo, macedone, bosniaco; Chekhia (traslitterato dal cirillico) in russo e in bulgaro; Çeki in albanese; Tjeckien in svedese; Tjekkiet in danese; Tjeckien in svedese; Tsjechië in olandese; ecc. Pure in Italia, peraltro, l'uso di Cechia è piuttosto diffuso, anche in sedi specializzate (come per esempio la "Rivista Italiana di Onomastica") ed è inoltre dominante nell'italiano della Svizzera. E, anzi, il numero delle pagine in Rete in cui occorre il sintagma "in Cechia" si equivale con le occorrenze dell'espressione "nella Repubblica Ceca". La forma corrispondente a Cechia, infine, esiste ed è usata anche in patria: Česko.

Le due indicazioni della nazione ceca hanno dunque pari dignità? La risposta sarebbe stata favorevole già negli anni scorsi; ma dal 2015 lo stesso governo ceco ha manifestato l'intenzione di semplificare il nome dello Stato, eliminando la voce republika, e il I4 aprile 2016 il presidente della Repubblica Miloš Zeman ha annunciato la presentazione alle Nazioni Unite della richiesta di cambiamento (in tutte le lingue del mondo e in particolare nelle situazioni in cui non è necessario utilizzare il nome formale: manifestazioni sportive, promozioni commerciali, ecc.).

Inutile sottolineare che anche in Cechia (localmente: Čsko) la proposta ha suscitato perplessità e forti opposizioni. Si sostiene, da una parte, che il coronimo Česko non è menzionato nella Costituzione ceca; ma, si replica dall'altra, neppure Československo (Cecoslovacchia) lo era e per esempio nella Costituzione della Repubblica slovacca non è mai indicata la voce d'uso corrente Slovakia. Ancora, chi avversa la sostituzione sostiene che Czechia sarebbe un neologismo: ma la prima attestazione in latino risale al I6oz e in inglese al I84I. Un nome scelto solo per la sua brevità non sarebbe necessario: si replica che un nome politico non può rimpiazzare un nome geografico, che una forma corta ufficiale rimpiazzerebbe le molte abbreviazioni errate o incomplete - in inglese Czecho, C. Rep., CR (che corrisponde ufficialmente alla Costa Rica, ecc.) e che "Made in Czech Republic" ha fallito come marchio nel mondo. Qualcuno porta l'esempio della Repubblica Dominicana e della Repubblica Centrafricana per dimostrare che l'accorciamento non è necessario: ma si tratta di due soli casi e, come visto, ben giustificati.

Si obietta ancora che le spese per il cambio ufficiale di nome sarebbero notevoli, ma si risponde che il mutamento sarebbe graduale e comunque Repubblica Ceca continuerebbe a figurare nei protocolli diplomatici nazionali e internazionali, nei trattati internazionali e in documenti analoghi. Si dice, ancora, che la voce Cechia, che propriamente sarebbe sinonimo di Boemia, escluderebbe la Moravia e la porzione della Slesia che sono parte dello Stato: ma la medesima obiezione vale per il nome attuale.

Si lamenta infine che Czechia sarebbe a rischio di confusione, ma si risponde che lo è semmai Czech, per l'analogia, almeno in inglese o in francese, con check e chéque, e la somiglianza con Cecenia non è maggiore di quanto non lo siano gli elementi delle coppie Iran-Iraq, Austria-Australia, Niger-Nigeria, Gambia-Zambia, per non dire della Georgia russa e della Georgia americana (per l'intero dibattito si può consultare, in inglese, il sito www.goczechia.com/).

Del resto le denominazioni ufficiali degli Stati sono spesso assai differenti - e più complesse - di quelle che anche nel registro ufficiale degli altri Paesi e nel linguaggio politico e giornalistico vengono regolarmente utilizzate: (quasi) nessuno oggi parla di Repubblica Islamica dell'Afghanistan, di Repubblica d'Albania, di Repubblica democratica e popolare di Algeria, di Repubblica d'Angola, di Repubblica Argentina, di Repubblica d'Armenia, di Repubblica d'Austria, di Repubblica d'Azerbaigian, per limitarsi alla lettera A-; oppure di Regno di Spagna, Regno Hashemita di Giordania, Confederazione Svizzera, Stato Plurinazionale di Bolivia, Ex Repubblica Jugoslava di Macedonia, Repubblica Orientale dell'Uruguay, Unione delle Comore, Gran Ducato di Lussemburgo, Stati Uniti Messicani, ecc. (le dizioni italiane sono la traduzione di quelle inglesi, francesi, spagnole, russe, arabe e cinesi pubblicate dall'UNGEGN, il Gruppo di esperti delle Nazioni Unite per i nomi geografici).

E senza andare lontano, anche l'Italia è ufficialmente "Repubblica Italiana". 


\section{Cita come:}

Enzo Caffarelli, Repubblica Ceca o Cechia?, "Italiano digitale", 2016, VIII, 2019/1 (gennaio-marzo), pp. 59-61.

DOI: $10.35948 / 2532-9006 / 2019.3082$

Copyright 2016 Accademia della Crusca

Pubblicato con licenza creative commons CC BY-NC-ND 